Case Report

\title{
Renal Tubular Acidosis in the Postpartum Period: A Case Report and Literature Review
}

\author{
Carlos E. Duran, ${ }^{1}$ Mayra Estacio ${ }^{D},{ }^{2}$ Fredy Lozano, ${ }^{3}$ Esteban Echeverri, ${ }^{4}$ \\ Maria Juliana Riascos, ${ }^{4}$ Juan Guillermo Posada, ${ }^{1}$ and Johanna Schweineberg ${ }^{1}$ \\ ${ }^{1}$ Department of Nephrology, Fundacion Valle del Lili, Cali, Colombia \\ ${ }^{2}$ Health Sciences Faculty, Universidad CES, Cali, Colombia \\ ${ }^{3}$ Health Sciences Faculty, Universidad del Valle, Cali, Colombia \\ ${ }^{4}$ Health Sciences Faculty, Universidad ICESI, Cali, Colombia
}

Correspondence should be addressed to Mayra Estacio; mayraestacio1@gmail.com

Received 19 November 2020; Revised 27 July 2021; Accepted 29 July 2021; Published 8 September 2021

Academic Editor: Yoshihide Fujigaki

Copyright (c) 2021 Carlos E. Duran et al. This is an open access article distributed under the Creative Commons Attribution License, which permits unrestricted use, distribution, and reproduction in any medium, provided the original work is properly cited.

\begin{abstract}
Case Presentation. Distal renal tubular acidosis (dRTA) is characterized by impaired hydrogen ion secretion in the distal nephron resulting either from decreased net activity of the proton pump or from increased luminal membrane hydrogen ion permeability. Typical complications of dRTA include severe hypokalemia, normal anion gap metabolic acidosis, nephrolithiasis, and nephrocalcinosis. The patient is a 25 -year-old woman in immediate puerperium with hypokalemia leading to paralysis, and the laboratory findings in this patients were concerning for dRTA. It is rare to encounter this entity during pregnancy, and the impact of this pathology is unknown.
\end{abstract}

\section{Case Presentation}

A 25-year-old woman in immediate puerperium was admitted to the hospital with proximal weakness that has been increasing progressively until difficulty in mobility and subsequent prostration that occurs seven hours after cesarean.

There were no personal or family history diseases, current medications include ferrous sulfate, and she denied alcohol or recreational drugs. She had one previous pregnancy without complication. This pregnancy was normal, and a cesarean section was performed at 37 weeks for pelvic dystocia.

On physical examination, she was alert and oriented, and her vital signs were normal in the neurological examination with findings of proximal weakness, without alteration in the tendon reflexes and findings of alteration in long tracts or focal neurologic deficit.

Initial laboratory showed normal blood cell counts, severe hypokalemia (potassium, $1.91 \mathrm{mEq} / \mathrm{L}$ ), and $\mathrm{pH}$ of
7.24, bicarbonate at $7.7 \mathrm{mmol} / \mathrm{L}$ with normal plasma anion gap (anion GAP: 12, $5 \mathrm{mEq} / \mathrm{L}$ ) Table 1.

Urine studies showed urinalysis was normal with a $\mathrm{pH}$ of 6.5, without urine active sediment. Urinary $\mathrm{K}: \mathrm{Cr} 83.7 \mathrm{mEq} / \mathrm{g}$, urinary anion gap: $24 \mathrm{mmol} / \mathrm{L}$, and urinary osmolar gap is positive Table 2.

Serologic testing was negative for autoimmune causes.

A renal ultrasound revealed normal-sized kidneys with marked increase in the echogenicity in the region of the renal pyramids bilaterally and symmetric consistent with nephrocalcinosis.

Treatment: She received potassium chloride initial by the peripheral vein, and 6 hours after admission, his serum potassium level increased to $2.1 \mathrm{mEq} / \mathrm{L}$ with improvement of weakness.

\section{Discussion}

The findings in this patients were concerning for dRTA complicated with severe symptomatic hypokalemia. Distal 
TABLE 1: Laboratory findings on admission.

\begin{tabular}{lcc}
\hline Parameter & Result & Reference range \\
\hline Sodium, mmol/L & 146.3 & $136-145$ \\
Potassium, mmol/L & 1.9 & $2.5-5.1$ \\
Chloride, mmol/L & 128.6 & $98-107$ \\
BUN, mg/dL & 8.9 & $6-20$ \\
Creatinine, mg/dL & 1.2 & $0.5-0.95$ \\
Calcium ionized, mmol/L & 1.43 & $1.15-1.33$ \\
Phosphorus, mg/dL & 2.44 & $2.5-4.5$ \\
Magnesium, mg/dL & 1.78 & $1.59-2.56$ \\
Arterial blood gas & & \\
pH & 7.249 & $7.35-7.45$ \\
PCO2, mm Hg & 17.9 & $35-45$ \\
PO2, mm Hg & 189.1 & $83-108$ \\
Bicarbonate, mEq/L & 7.7 & $21-28$ \\
Osmalality, mOsm/kgH20 & 313 & $275-300$ \\
\hline
\end{tabular}

TABle 2: Urine studies.

\begin{tabular}{lc}
\hline Parameter & Result \\
\hline Spot urine chemistry & \\
Osmolality, mOsm/kg H20 & 325 \\
Sodium, mmol/L & 113.5 \\
Potassium, mmol/L & 17.59 \\
Chloride, mmol/L & 106.3 \\
Creatinine, mg/dL & 21.13 \\
Urea nitrogen, mg/dL & 169.30 \\
24-hour urine chemistry & \\
Volume, mL/24h & 4244 \\
Sodium, mEq/24h & 447.74 \\
Potassium, mEq/24h & 80.51 \\
Chloride, mEq/24h & 451.14 \\
Calcium, mg/24h & 273.74 \\
Magnesium, mg/24h & 87.4 \\
\hline
\end{tabular}

RTA, also known as type 1 RTA, is characterized by impaired hydrogen ion secretion in the distal nephron for a decreased net activity of the proton pump or increased hydrogen ion permeability of the luminal membrane [1].

The distal renal tubular acidosis should be considered in any patient with an otherwise unexplained normal anion gap metabolic acidosis and hypokalemia. The potassium wasting is generated by the reduction in distal hydrogen ion secretion via several mechanisms such as increase in aldosterone levels due to sodium wasting, as well as metabolic acidosis $[1,2]$.

This pathology can be associated with nephrocalcinosis, urinary calculi, citraturia, calciuria, osteopenia, osteomalacia, and secondary hyperparathyroidism. It can be seen in the context of immunologic diseases such as Sjögren, systemic lupus erythematosus and rheumatoid arthritis, medications (ifosfamide, amphotericin B, lithium carbonate, and ibuprofen), hypercalciuric conditions (hyperparathyroidism, vitamin D intoxication, and sarcoidosis), and others, including familial causes (medullary sponge kidney and Wilson's disease) $[1,2]$.

During pregnancy, there are alterations in the way the kidney manages solutes and filtration of solutes looking for an optimal environment for fetal development. The glomerular filtration rate (GFR) increases about $40-50 \%$ by week 9 [3]. There is an increase in the urea excretion and, in some cases, variable glycosuria due to a diminished glucoseabsorption capacity in the distal tubule. Additionally, lowgrade-level proteinuria that generally will not exceed $300 \mathrm{mg} / 24 \mathrm{~h}$ is observed [3]. There is also a change in the osmostat, which leads to a decrease in the normal osmolality to $270 \mathrm{mOsm} / \mathrm{kg}$, at which level the secretion of antidiuretic hormone would start, with a concomitant reduction of sodium level of around 4 to $5 \mathrm{mEq} / \mathrm{L}$ in normal pregnancy.

Pregnancy is a vasodilated state with an important grade of water retention which also contributes to the plasma sodium reduction [3]. Additionally, in most pregnancies, the rate of potassium excretion is constant with changes in tubular reabsorption that adapt to the GFR [3]. This is accompanied by a physiological compensated respiratory alkalosis due to hyperventilation which is asymptomatic, but poses a disadvantage if metabolic acidosis ensues [4].

The presence of hypokalemia in pregnancy is unusual. There are reported cases of severe hypokalemia in pregnancy associated with renal tubular acidosis in patients with a diagnosis before the pregnancy [5-7]. The impact of dRTA for the mother and child is unknown. The physiological changes in pregnancy could worsen IRTA, and it is believed that due to the increase in GFR during pregnancy, this can lead to an increase of electrolyte loss in urine, including potassium. Women without tubular alterations should be capable of dealing with the increased demand for potassium absorption in the distal tubule. Yet, if symptomatic or asymptomatic alterations exist, the state of increased renal and metabolic demand can overcome the renal potassium absorption renal capacity and lead to an increased kaliuresis and concomitant symptomatic hypokalemia [8]. Additionally, the potassium loss can be understood as a compensatory mechanism for the metabolic acidosis generated by the renal distal tubular acidosis [8].

All cases of hypokalemia in pregnant patients and during the puerperium should be investigated making emphasis in the acid-base balance, the renin/aldosterone axis, and in identifying renal or extrarenal potassium losses. Additionally, the use of certain medications such as steroids, diuretics, insulins, and beta-agonists should be discarded [9]. There are also reports of renal proximal tubular acidosis during pregnancy associated with toluene intoxication due to inhalants abuse, so drug use must always be questioned [5].

Distal tubular acidosis in pregnancy is rare. In some cases, the acid-base and potassium status during follow-up will normalize [5], so it is important to obtain serial potassium and blood gas measurements to determine their behavior in this patient. Generally, these patients are treated with bicarbonate supplementation, potassium citrate, and education for an increased awareness of the delicate and unstable balance of acid-base and potassium homeostasis in people living with renal tubular acidosis.

\section{Data Availability}

The data used to support the findings of this study are included within the article. 


\section{Conflicts of Interest}

The authors declare no conflicts of interest.

\section{References}

[1] R. Mustaqeem and A. Arif, Renal Tubular Acidosis, StatPearls Publishing, Treasure Island, FL, USA, 2020, http://www.ncbi. nlm.nih.gov/pubmed/30085586.

[2] M. Soleimani and A. Rastegar, "Pathophysiology of renal tubular acidosis: core curriculum 2016," American Journal of Kidney Diseases, vol. 68, no. 3, pp. 488-498, 2016.

[3] K. L. Cheung and R. A. Lafayette, "Renal physiology of pregnancy," Advances in Chronic Kidney Disease, vol. 20, no. 3, pp. 209-214, 2013.

[4] V. Lim, A. Katz, and M. Lindheimer, "Acid-base regulation in pregnancy," American Journal of Physiology-Legacy Content, vol. 231, no. 6, pp. 1764-1769, 1976.

[5] D. Narcisse, M. Agarwal, and A. Kumar, "A rare case of transient proximal renal tubular acidosis in pregnancy," Case Reports in Nephrology, vol. 2017, Article ID 1342135, 4 pages, 2017.

[6] H. Seeger, P. Salfeld, R. Eisel, C. A. Wagner, and N. Mohebbi, "Complicated pregnancies in inherited distal renal tubular acidosis: importance of acid-base balance," Journal of $\mathrm{Ne}$ phrology, vol. 3, 2017.

[7] O. Fikri Benbrahim, F. Cazalla Cadenas, A. Valentín Martín, and E. D. Valladares Molleda, "Acidosis tubular renal distal en una gestante de siete semanas: diagnóstico, complicaciones y tratamiento," Nefrologia, vol. 31, no. 6, pp. 761-763, 2011.

[8] C. J. Firmin, T. F. Kruger, and R. Davids, "Proximal renal tubular acidosis in pregnancy: a case report and literature review," Gynecologic and Obstetric Investigation, vol. 63, no. 1, pp. 39-44, 2007.

[9] M. Kulkarni, T. V. Srividya, and N. Gopal, "Hypokalemia paraplegia in pregnancy," Journal of Clinical Diagnostic Research, vol. 8, no. 6, pp. 13-14, 2014. 